\title{
5 Research Square

\section{Premature Ovarian Insufficiency After Bone Marrow Transplantation for Thalassemia Major in a Patient With Primary Amenorrhea and 46, XY Karyotype: a Case Report and Literature Review}

Hui Li

Shenzhen Baoan Women's and Children's Hospital

Jin Li

Shenzhen Baoan Women's and Children's Hospital

Xiaohong Li

Shenzhen Baoan Women's and Children's Hospital

Hong Yi

Shenzhen Baoan Women's and Children's Hospital

Qixiu Ren

Shenzhen Baoan Women's and Children's Hospital

Xiaoyan Chen ( $\nabla$ chenxiaoyan@cuhk.edu.hk)

Shenzhen Baoan Women's and Children's Hospital

\section{Case report}

Keywords: latrogenic factor, Premature ovarian insufficiency, bone marrow transplantation, XY karyotype, case report

Posted Date: January 28th, 2021

DOl: https://doi.org/10.21203/rs.3.rs-154411/v1

License: (c) (i) This work is licensed under a Creative Commons Attribution 4.0 International License. Read Full License 


\section{Abstract}

Background: latrogenic factor is one of the recognized causes for premature ovarian insufficiency. The aim of this case report was to present a rare case with premature ovarian insufficiency and $46, X Y$ karyotype after bone marrow transplant for thalassaemia major at childhood. We also reviewed some relevant literature in this report.

Case presentation: A 17-year-old girl was presented with primary amenorrhea and premature ovarian insufficiency after receiving chemotherapy and bone marrow therapy from her brother due to thalassaemia major at childhood. She had poor secondary sex characteristics, assessed as stage I for the development of breasts and external genitalia based on the Tanner scale. Transabdominal ultrasound showed small uterus with visible endometrial lining and small ovaries. Laboratory data showed hypergonadotropic hypogonadism profile with low level of estrogen and high level of follicularstimulating hormone (FSH). Patient's peripheral lymphocytes karyotype was $46, \mathrm{XY}$.

Conclusions: A correct diagnosis is not only dependent on clinical manifestations, but also the detailed medical history. In light of the relatively high prevalence of thalassemia, fertility preservation should be considered for young girls before they receive chemotherapy, radiation treatment and bone marrow transplant.

\section{Background}

Premature ovarian insufficiency affects around $1 \%$ of women under the age of 40 . It usually presents as oligo- or amenorrhea for at least four months and FSH level over $40 \mathrm{IU} / \mathrm{ml}$, checked twice at least four weeks apart. ${ }^{1,2}$ When serious ovarian function depletion occurs in childhood, most patients will experience primary amenorrhea.

latrogenic factors are one of the recognized causes for premature ovarian insufficiency, including radiation treatment and cheomotherapy. The use of chemotherapy, radiotherapy and bone marrow transplantation (BMT) to treat malignant and nonmalignant diseases in girls and young women has been more and more common. ${ }^{3}$ Therefore, the awareness of fertility preservation for this group of subjects should be emphasized since the late side effects of BMT though usually not life threatening, may significantly impair quality of life in adults, ${ }^{3}$ in whom gonadal failure is a common long-term endocrine consequence of BMT.

In this case report, we present a patient with primary amenorrhea and premature ovarian insufficiency after chemotherapy and BMT due to thalassaemia major, whose peripheral lymphocytes karyotype result $(46, X Y)$ could lead to a misdiagnosis of disorders of sexual differentiation.

\section{Case Presentation}


This report was approved the local hospital Ethics Committee. A 17-year-old girl attended our outpatient clinic for evaluation of primary amenorrhea. Her past medical history revealed short period of chemotherapy followed by BMT therapy due to thalassaemia major at the age of eight. She reported to

be free of the disease currently and did not take any medication. As an adolescent, the patient came with her mother, reported a lack of menstrual flow and was presented with poor secondary sex characteristics.

During the visits, she was assessed as stage I for the development of breasts and external genitalia according to the Tanner scale. The presence of vaginal opening was found by gynecological examination. Transabdominal ultrasound showed small uterus $(2.1 \times 1.5 \times 1.0 \mathrm{~cm})$ with visible endometrial lining; both ovaries were measured as $1.6 \times 0.8 \mathrm{~cm}$ (Fig. 1). Laboratory data showed low concentration of estradiol ( $<55 \mathrm{pmol} / \mathrm{l})$ and significantly elevated levels of serum follicle-stimulating hormone (FSH) and luteinizing hormone (LH) $(125.93 \mathrm{mIU} / \mathrm{ml}$ and $31.59 \mathrm{mlU} / \mathrm{ml}$, respectively). Anti-Mullerian hormone (AMH) concentration was extremely low $(0.04 \mathrm{ng} / \mathrm{ml})$. The rest of the results including levels of basal thyroid hormones, TSH, cortisol, 17-hydroxyprogesterone and prolactin were within the reference range. X-ray of the left wrist showed that the bone age was 13 years old (Fig. 2). Patient's peripheral lymphocytes karyotype was $46, \mathrm{XY}$. A more detailed history was then taken and it was found that the patient had BMT from her brother. Based on the above characteristics, the patient was diagnosed as primary amenorrhea and premature ovarian insufficiency.

Currently, the patient receives low dose of estrogen therapy ( $0.25 \mathrm{mg}$ estradiol valerate orally daily) to allow the growth and development of breast and reproductive organs as well as the skeleton. The patient will be followed-up every 3 months for regular checkups in our outpatient clinic. When there is improvement of the uterus size and endometrial thickness, hormone replacement treatment using estradiol and norgestrel may be given to induce menstruation.

\section{Discussion And Conclusion}

In females, menarche is the most significant corporal change during adolescence. Primary amenorrhea is defined as the absence of menstruation by the age of 15 with developed secondary sexual characteristics or three year after thelarche. ${ }^{4}$ There are several classifications of causes of primary amenorrhea, including anatomic defects, primary hypogonadism, hypothalamic causes, pituitary causes, chromosomal abnormalities and other endocrine gland disorders. ${ }^{5}$

The prevalence of $46, X Y$ karyotype in females with primary amenorrhea, either with pure gonadal dysgenesis or complete androgen insensitivity syndrome is around $3-6 \%{ }^{6}$ Pure gonadal dysgenesis, also known as Swyer syndrome, may be recognized when there are not normally differentiated testes despite male karyotype. It has been reported that the mutation in several genes are responsible for Swyer syndrome, among which SRY appears to be the most significant one and responsible for $10-20 \%$ of cases. ${ }^{7}$ Patients with Swyer syndrome are typically tall-statured and with female genitalia. Another feature is hypergonadotropic hypogonadism with low levels of estrogen and $\mathrm{AMH}$ and high level of FSH, which results in poor breast development, amenorrhea, increased risk of cardiovascular disease. ${ }^{7}$ With 
regards to complete androgen insensitivity syndrome, tissues are not reactive to testosterone due to the mutation in gene encoding androgen receptor, although testes are developed and their hormone secretion function is preserved. Affected individuals are with the external female phenotype and genitalia due to the normal conversion of excessive testosterone to estrogen. However, Mullerian derivatives are not preserved and testes may be situated either in the abdominal cavity or throughout the inguinal canal or in the labia majora. Hormonal profiles usually show elevated testosterone and detectable estrogen concentrations. $^{8}$

The clinical features in our patient presented in this report, including female external genitalia, presence of uterus, hypergonadotropic hypogonadism, low AMH level and 46, XY karyotype can be easily misdiagnosed as Swyer syndrome. However, when digging the past history of the patient, it was found that the appearance of $46, X Y$ karyotype in the peripheral blood in this patient is due to the bone marrow transplant from a male donor. This clue can help to rule out the diagnosis of Swyer syndrome. There are two similar cases reported previously. One study reported a childhood cancer survivor with premature ovarian insufficiency and $46 \mathrm{XY}$ karyotype in lymphocytes after chemotherapy and BMT from an unrelated male donor, ${ }^{9}$ and the other report showed a patient with primary amenorrhea and $46 \mathrm{XY}$ karyotype after receiving BMT from her brother and chemotherapy due to acute myeloid leukemia. ${ }^{10}$ The authors from both reports also found a high likelihood of misdiagnosis of Swyer syndrome, which was similar to our report. Therefore, the comprehensive collection of detailed present and past medical history is pretty important for a correct diagnosis.

In this case presented, several possible causes may be contributing to the observed iatrogenic premature ovarian insufficiency. After repeated red blood cell transfusions, patients with thalassemia major may have ovarian impairment due to iron overload, when the transferrin-dependent system is inhibited through ferritin saturation pathway and excessive iron accumulation occurs through the non-transferrin bound iron (NTBI) pathway. ${ }^{11}$ Although the most common endocrinopathy in patients with thalassemia is the hypogonadism resulting from iron deposition in the hypothalamic and/or pituitary cells, ${ }^{12}$ it is also worth noting that iron overload may affect ovarian function directly as well. An earlier study has shown an inverse correlation between $\mathrm{AMH}$ level and $\mathrm{NTBI},{ }^{13}$ suggesting suspected ovarian tissue iron overload in women with thalassemia major. Another study also demonstrated that AMH level and antral follicle count are significantly decreased in women with transfusion-dependent thalassemia major compared with agematched controls. ${ }^{12}$ These findings support a deleterious effect of iron overload on ovarian tissue, which may result in an increase in reactive oxygen species and the subsequent acceleration in follicular aging. ${ }^{14}$ An earlier study has found high redox activity in the ovarian follicular fluid from a woman with thalassemia major, which suggested that redox-active iron ions may mediate free radical production and induce ovarian tissue injury. ${ }^{15}$ Therefore, there is a need to better define the appropriate chelation regimens and antioxidant supplementations regarding reproductive function in women with thalassemia major receiving blood transfusion treatment. However, whether ovarian function is impaired by a direct effect of iron overload is not clearly understood yet. 
Another important factor responsible for the ovarian impairment in this case may be BMT, including chemotherapy and/or total body irradiation (TBI). The extent of follicular impairment can be affected by the type of TBI protocol as well as the age receiving BMT. In terms of chemotherapy, busulfan appears to be the most gonadotoxic regimen, with reported prevalence of premature ovarian failure as high as $100 \%{ }^{16,17}$ Low-dose cyclophosphamide $(200 \mathrm{mg} / \mathrm{kg})$ is considered to be much less gonadotoxic compared with other regimens, with good recovery rates of clinical ovarian function, particularly in women younger than 25 years old. ${ }^{18,19}$ In contrast, TBI appears to be a more toxic treatment prior to BMT and most patients undergoing TBI experience gonadal failure. Compared with TBI administration after puberty, TBI administered prior to puberty is reported to be less gonadotoxic and around $40-60 \%$ of patients experienced apontaneous puberty. ${ }^{20,21}$ This might be due to the higher number of non-growing follicles found in younger girls and some other particular anatomical or paracrine factors, which might lead to a higher resistance to fibosis implicated in the mechanisms of ovarian damage. ${ }^{22}$

To conclude, this is a case report of a patient presenting with primary amenorrhea, premature ovarian insufficiency and 46, XY karyotype in peripheral blood after receiving chemotherapy and BMT from her brother in childhood due to thalassemia major. It is important that gynecologists should be aware that not only the clinical manifestations, but also the detailed medical history are crucial for a correct diagnosis and the following treatment. In light of the relatively high prevalence of thalassemia, particularly in the southern China, protection of ovarian function should be considered for young girls who are going to have chemotherapy, total body irradiation and BMT.

\section{Declarations}

\section{Acknowledgements}

Not applicable.

\section{Authors' contributions}

Hui Li, Jin Li and Xiaoyan Chen: Substantial contribution to the conception and design of the work; Hui Li, Xiaohong Li, Hong Yi and Qixiu Ren: performed investigations on the patient; Hui Li and Xiaoyan: participation in acquisition of the literature; Hui Li, Jin Li and Xiaoyan Chen: manuscript drafting. All authors have read and approved the final article.

\section{Funding}

This study was supported by Shenzhen Key Medical Discipline Construction Fund (SZXK028)

\section{Availability of data and materials}

The data used or analyzed are all included in this report.

\section{Ethics approval and consent to participate}


This paper was approved by the Ethics Committee of the institutional review board (IRB) of Shenzhen Baoan Woman's and Children's Hospital and a written informed consent has been obtained from the patient.

\section{Consent for publication}

Written informed consent was obtained from the patient for the publication of this case report and any accompanying images. A copy of the written consent is available on request.

\section{Competing interests}

The authors declare that they have no competing interests.

\section{References}

1. Coulam C, Adamson S and Annegers J. Incidence of premature ovarian failure. Obstet Gynecol 1986; 67: 604-06.

2. De Vos M, Devroey P and Fauser BC. Primary ovarian insufficiency. 2010 11;376(9744):911-21.

3. Donnez J and Dolmans MM. Fertility preservation in women. Nat Rev Endocrinol. 2013;9(12):735-49.

4. Teede HJ, Misso ML, Costello MF, et al. International PCOS Network. Recommendations from the international evidence-based guideline for the assessment and management of polycystic ovary syndrome. Fertil Steril. 2018;110(3):364-379.

5. Practice Committee of American Society for Reproductive Medicine. Current evaluation of amenorrhea. Fertil Steril. 2008;90(5Suppl):S219-25.

6. Kriplani A, Goyal M, Kachhawa G, et al. Etiology and management of primary amenorrhoea: A study of 102 cases at tertiary centre. Taiwan J Obstet Gynecol. 2017 Dec;56(6):761-764. DOI: 10.1016/j.tjog.2017.10.010. PMID: 29241916.

7. King TF and Conway GS. Swyer syndrome. Curr Opin Endocrinol Diabetes Obes. 2014;21(6):504-10.

8. Michala L and Creighton SM. The XY female. Best Pract Res Clin Obstet Gynaecol. 2010;24(2):13948.

9. Kruszewska J, Krzywdzińska S, Grymowicz M, et al. POI after chemotherapy and bone marrow transplant may mimic disorders of sexual differentiation - a case report of a patient with primary amenorrhea and 46, XY karyotype. Gynecol Endocrinol. 2020;36(6):564-566.

10. Huang $\mathrm{H}$ and Tian Q. Primary amenorrhea after bone marrow transplantation and adjuvant chemotherapy misdiagnosed as disorder of sex development: A case report. Medicine (Baltimore). 2016;95(44):e5190.

11. Hershko C, Link G and Cabantchik I. Pathophysiology of iron overload. Ann N Y Acad Sci. 1998;850:191-201.

12. Uysal A, Alkan G, Kurtoğlu A, et al. Diminished ovarian reserve in women with transfusion-dependent beta-thalassemia major: Is iron gonadotoxic? Eur J Obstet Gynecol Reprod Biol. 2017;216:69-73. 
13. Singer ST, Vichinsky EP, Gildengorin G, et al. Reproductive capacity in iron overloaded women with thalassemia major. 2011;118(10):2878-81.

14. Kitajima M, Defrère $S$, Dolmans MM, et al. Endometriomas as a possible cause of reduced ovarian reserve in women with endometriosis. Fertil Steril. 2011;96(3):685-91.

15. Schubert B, Canis M, Darcha $C$, et al. Human ovarian tissue from cortex surrounding benign cysts: a model to study ovarian tissue cryopreservation. Hum Reprod. 2005;20(7):1786-92.

16. Grigg AP, McLachlan R, Zaja J, et al. Reproductive status in long-term bone marrow transplant survivors receiving busulfan-cyclophosphamide (120 mg/kg). Bone Marrow Transplant. 2000;26(10):1089-95.

17. López-lbor B and Schwartz AD. Gonadal failure following busulfan therapy in an adolescent girl. Am J Pediatr Hematol Oncol. 1986;8(1):85-7.

18. Nabhan SK, Bitencourt MA, Duval M, et al. Fertility recovery and pregnancy after allogeneic hematopoietic stem cell transplantation in Fanconi anemia patients. Haematologica. 2010;95(10):1783-7.

19. Sanders JE, Buckner CD, Amos D, et al. Ovarian function following marrow transplantation for aplastic anemia or leukemia. J Clin Oncol. 1988;6(5):813-8. .

20. Borgmann-Staudt A, Rendtorff R, Reinmuth $S$, et al. Fertility after allogeneic haematopoietic stem cell transplantation in childhood and adolescence. Bone Marrow Transplant. 2012;47(2):271-6.

21. Sanders JE. The impact of marrow transplant preparative regimens on subsequent growth and development. The Seattle Marrow Transplant Team. Semin Hematol. 1991;28(3):244-9.

22. Meirow D, Biederman $H$, Anderson RA, et al. Toxicity of chemotherapy and radiation on female reproduction. Clin Obstet Gynecol. 2010;53(4):727-39.

\section{Figures}




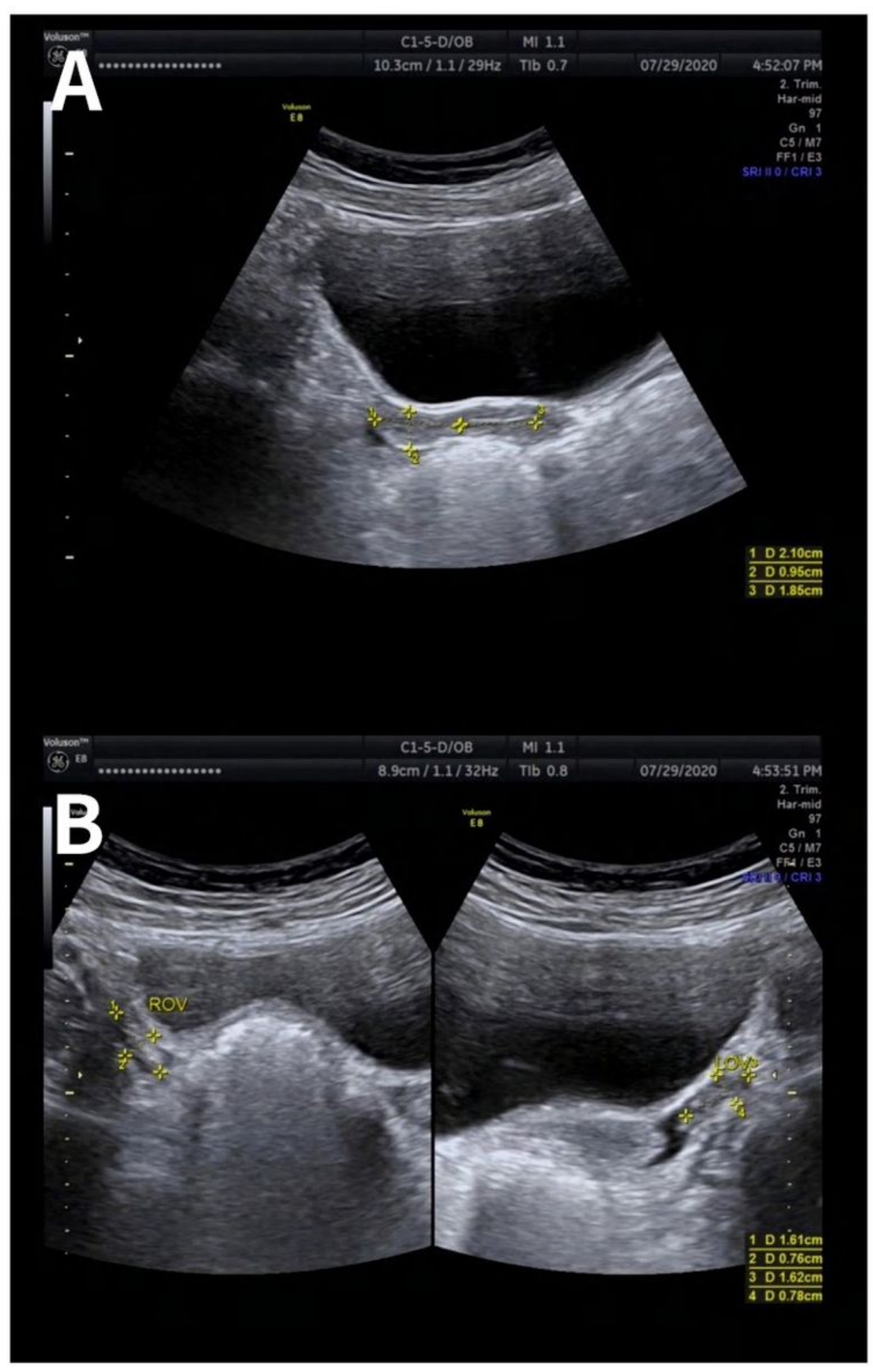

\section{Figure 1}

Ultrasonography image of uterus and ovaries. Transabdominal ultrasound showed small uterus $(2.1 \times 1.5 \times 1.0 \mathrm{~cm})$ with visible endometrial lining $(A)$. Both ovaries were measured as $1.6 \times 0.8 \mathrm{~cm}(B)$. 


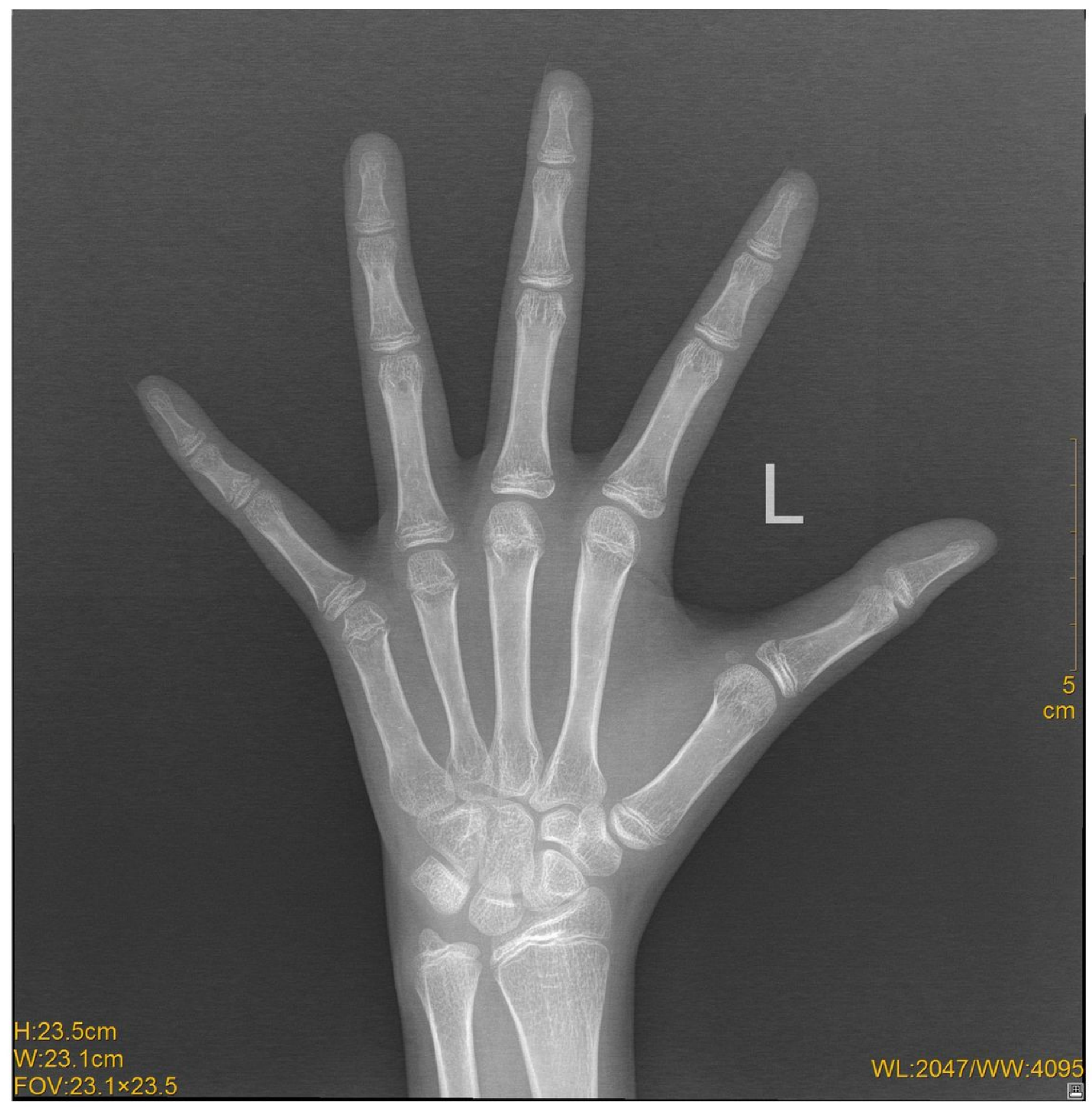

\section{Figure 2}

X-ray image of the left wrist. X-ray showed that the bone age was 13 years old. 